Association for Information Systems

AIS Electronic Library (AISeL)

ICIS 2021 TREOS

TREO Papers

$12-12-2021$

\title{
Evolution of scholarly collaboration in ICIS: a scientometric analysis
}

Yuming $\mathrm{He}$

Old Dominion University, yhe004@odu.edu

Lingyu Li

Old Dominion University, Ili017@odu.edu

Yifang Ma

yma004@odu.edu

Weiru Chen

Old Dominion University, wchen011@odu.edu

Follow this and additional works at: https://aisel.aisnet.org/treos_icis2021

\section{Recommended Citation}

He, Yuming; Li, Lingyu; Ma, Yifang; and Chen, Weiru, "Evolution of scholarly collaboration in ICIS: a scientometric analysis" (2021). ICIS 2021 TREOs. 8.

https://aisel.aisnet.org/treos_icis2021/8

This material is brought to you by the TREO Papers at AIS Electronic Library (AISeL). It has been accepted for inclusion in ICIS 2021 TREOs by an authorized administrator of AIS Electronic Library (AISeL). For more information, please contact elibrary@aisnet.org. 
TREO

Technology, Research, Education, Opinion

\section{Evolution of scholarly collaboration in ICIS: a scientometric analysis}

Yuming He, yhe004@odu.edu; Lingyu Li, lli017@odu.edu; Yifang Ma, yma004@odu.edu; Weiru Chen, wchen011@odu.edu

Information systems research is multidisciplinary, with close connections to computer science, psychology, sociology, economics, and other business disciplines(Banville and Landry 1989). Understanding the social dynamics of a research community is helpful to assess a scientific discipline and evaluate its status and progress(Abbasi, Altmann, et al. 2011). In a research community, stakeholders interact with each other by sharing common research interests and ideas, research methods, and techniques and influence each other's work(Culnan 1986). These social interactions interweave a complex social network in which knowledge is generated, disseminated, and updated between researchers.

To foster the growth of multidiscipline, collaboration is one of the most effective ways (Oh et al. 2005). As the Information Systems (IS) community grows, it is reasonable to expect that collaborations between scholars have increased. we studies the evolution and trend of collaboration networks in the International Conference on Information Systems (ICIS). Based on evolutionary analysis of papers published in ICIS proceedings over the 40 years from 1980 to 2020 , various characteristics of the social network of IS researchers are identified and discussed. Our research shows that key characteristics of the research collaboration network as reflected by the co-authorship: 1) ICIS community has thrived that demonstrated frequent interactions among members as new members have joined and connections between members have improved. 2) The critical core of the community, the most productive authors and countries, were identified. 3) Collaboration patterns of the community were also studied. 4) how social networks evolved was revealed.

\section{References}

Abbasi, A., Altmann, J., and Hossain, L. 2011. "Identifying the Effects of Co-Authorship Networks on the Performance of Scholars: A Correlation and Regression Analysis of Performance Measures and Social Network Analysis Measures," Journal of Informetrics (5:4), Elsevier, pp. 594-607. (https://doi.org/10/btk4xp).

Banville, C., and Landry, M. 1989. "Can the Field of MIS Be Disciplined?," Communications of the ACM (32:1), ACM New York, NY, USA, pp. 48-6o. (https://doi.org/10/c345sg).

Culnan, M. J. 1986. “The Intellectual Development of Management Information Systems, 1972-1982: A Co-Citation Analysis,” Management Science (32:2), INFORMS, pp. 156-172. (https://doi.org/10/ddkskn). 